\title{
Mixture of Two Inverse Exponential Distributions Based on Fuzzy Data
}

\author{
Shurooq A. K. Al-Sultany ${ }^{1 *}$ and Sahar Ahmed Mohammed ${ }^{2}$ \\ ${ }^{1}$ Department of Mathematics, College of Science, Mustansiriyah University, Iraq \\ ${ }^{2}$ Lecturer in the Department of Mathematics, College of Science, Mustansiriyah University, Iraq \\ *Corresponding Author: Shuroq80@yahoo.com
}

\begin{abstract}
In this paper we will discuss the mixture distribution consisting of two Inverse Exponential Distributions (MTIED) based on fuzzy data. We will study the Maximum Likelihood Estimator (MLE) via the Newton Raphson (NR) algorithm and Bayes estimation under square error loss and quadratic loss functions for the unknown parameters of the distribution, and reliability function. The obtained estimates of the unknown parameters and reliability function are compared numerically through Monte-Carlo simulation study in term of the mean square error (MSE) values and (IMSE) respectively. [DOI: 10.22401/ANJS.00.2.11]
\end{abstract}

Keywords: Finite Mixtures; Reliability; Inverse Exponential distribution; NR algorithm; Lindley's approximation; Mean square error; quadratic loss function.

\section{Introduction}

Finite mixture models play an important role in many applicable fields, such as economics, medicine, psychology, life testing, reliability analysis and etc., [12]. Several researches have assumed that the underlying population is a homogeneous one with the failure time distribution given by $F(x, \theta)$, where the form $\mathrm{F}$ is known but the parameter $\theta$ is unknown, [11].

Many authors interested with inferences on mixtures of exponential distributions and among them Jaheen [7] and Everitt and Hand [6]. Also, Elsherpieny [5] estimated the parameters of mixed generalized exponentially distributions.

The past experience as well as experimental constraints may suggest that the assumption of homogeneity may not hold and the underlying population may consist of several subpopulation, say $s p_{1}, s p_{2}, \ldots, s p_{k}$ mixed in proportion; $p_{1}, p_{2}, \ldots, p_{k}$.

Further, cumulative distribution function in each subpopulation is given by $F_{j}\left(x, \theta_{j}\right), j=1, \ldots, k$ with p.d.f.'s $f_{j}\left(x, \theta_{j}\right)$ respectively [10].

Inverse Exponential distribution is also known as reciprocal exponential distribution finds use in the analysis of fading wireless communication systems.

The mixture of two Inverse Exponential distributions (MTIES) has its p.d.f. as:

$f\left(x ; \theta_{1}, \theta_{2}\right)=p_{1} f_{1}\left(x ; \theta_{1}\right)+p_{2} f_{2}\left(x ; \theta_{2}\right) .$. $p_{1}+p_{2}=1,0<p_{1}, p_{2}<1$, (i.e.,$k=2$ )

where $f_{i}\left(x ; \theta_{i}\right)$, the density function of the $\mathrm{i}^{\text {th }}$ component (inverse exponential), is given by:

$f_{i}\left(x, \theta_{i}\right)=\frac{\theta_{i}}{x^{2}} e^{-\theta_{i / x}}, x>0, \theta_{i}>0, i=1,2$

The cumulative distribution function (CDF) of the MTIED is given by:

$F\left(x ; \theta_{1}, \theta_{2}\right)=p_{1} F_{1}\left(x, \theta_{1}\right)+p_{2} F_{2}\left(x ; \theta_{2}\right)$

where $F_{i}\left(t, \theta_{i}\right)$, the c.d.f. of the $\mathrm{i}^{\text {th }}$ component, is given by:

$F_{i}\left(x ; \theta_{i}\right)=e^{-\theta_{i / x}}, x>0, \theta_{i}>0, i=1,2$

The reliability function at time $t$ is given by:

$R(t)=p_{1}\left(1-e^{-\theta_{1} / t}\right)+p_{2}\left(1-e^{-\theta_{2} / t}\right), t \geq 0$

Note that the mean of the p.d.f. of the MTIED given in (1) and (2) does not exist. The inverse Generalized Exponential and the inverse Weibull distributions are both the generalization of an inverse exponential distribution [8].

Usually, it is assumed that an observed data are precise (exact) numbers. However, in real world situations, some collected data might be imprecise and are represented in the form of fuzzy numbers. The first publications in fuzzy set theory by Zadeh [13].

Thus, this paper focused on evaluate the estimating the unknown parameters and reliability function of MTIED through the methods (MLE, Bayes) based on fuzzy data 
and presenting a comparative study for estimating.

\section{Maximum Likelihood Estimators}

Let $\underline{x}=\left(x_{1}, x_{2}, \ldots, x_{n}\right)$ be an i.i.d. random vector of a random sample of size $n$ from MTIED with p.d.f. given by (1).If a realization of $\underline{x}$ was known exactly, then the complete data likelihood function is:

$$
\begin{aligned}
& L\left(\theta_{1}, \theta_{2} \mid \underline{x}\right)=\prod_{i=1}^{n} f_{x}\left(x_{i}, \theta_{1}, \theta_{2}\right) \\
& \quad=\prod_{i=1}^{n}\left[\frac{p_{1} \theta_{1}}{x^{2}} e^{-\theta_{1} / x}+\frac{\left(1-p_{1}\right) \theta_{2}}{x^{2}} e^{-\theta_{2 / x}}\right]
\end{aligned}
$$

Now, suppose that $x$ is not observed precisely and only partial informations about $\underline{x}$ are available in the form of fuzzy subset $\underline{\tilde{x}}$ with the Borel measurable membership function $\mu_{\tilde{x}}(x)$, so we can compute its probability according to Zadeh's definition of the probability of a fuzzy event $\tilde{A}$ in $R^{n}$, which is defined as the expectation of the membership function $\mu_{\tilde{A}}$ with respect to $\mathrm{p}$, [14]:

$P(\tilde{A})=\int \mu_{\tilde{A}}(x) d p ; \forall x \in R^{n}$

The observed-data likelihood function can then be obtained as:

$$
\begin{array}{r}
L\left(\theta_{1}, \theta_{2} \mid \underline{\tilde{x}}\right)=\prod_{i=1}^{n} \int\left[\frac{p_{1} \theta_{1}}{x^{2}} e^{-\theta_{1} / x}+\right. \\
\left.\frac{\left(1-p_{1}\right) \theta_{2}}{x^{2}} e^{-\theta_{2} / x}\right] \mu_{\widetilde{x_{l}}}(x) d x
\end{array}
$$

and the observed -data natural log-likelihood function will be:

$$
\begin{array}{r}
\ln L\left(\theta_{1}, \theta_{2} \mid \underline{\tilde{x}}\right)=\sum_{i=1}^{n} \ln \int\left[\frac{p_{1} \theta_{1}}{x^{2}} e^{-\theta_{1} / x}+\right. \\
\left.\frac{\left(1-p_{1}\right) \theta_{2}}{x^{2}} e^{-\theta_{2} / x}\right] \mu_{\widetilde{x}_{l}}(x) d x . .(8)
\end{array}
$$

assuming that the parameters $\theta_{1}$ and $\theta_{2}$ are unknown and $\mathrm{P}$ is known.

Differentiating the natural Log-likelihood function $\ell\left(\theta_{1}, \theta_{2} \mid \underline{\tilde{x}}\right)$, given by equation (8), partially with respect to $\theta_{1}, \theta_{2}$ and then equating to zero, we have:

$$
\begin{aligned}
& \frac{\partial \ln L\left(\theta_{1}, \theta_{2} \mid \underline{\tilde{x}}\right)}{\partial \theta_{1}}=\sum_{i=1}^{n} \frac{\int\left[\frac{p_{1}}{x^{2}} e^{-\theta_{1} / x}-\frac{p_{1} \theta_{1}}{x^{3}} e^{-\theta_{1} / x}\right] \mu_{\widetilde{x_{l}}}(x) d x}{\int\left[\frac{p_{1} \theta_{1}}{x^{2}} e^{-\theta_{1} / x}+\frac{\left(1-p_{1}\right) \theta_{2}}{x^{2}} e^{-\theta_{2} / x}\right] \mu_{\widetilde{x_{l}}}(x) d x}=0 \\
& \frac{\partial \ln L\left(\theta_{1}, \theta_{2} \mid \underline{\tilde{x}}\right)}{\partial \theta_{2}}=\sum_{i=1}^{n} \frac{\int\left[\frac{\left(1-p_{1}\right)}{x^{2}} e^{-\theta_{2} / x}-\frac{\left(1-p_{1}\right) \theta_{2}}{x^{3}} e^{-\theta_{2} / x}\right] \mu_{\widetilde{x_{l}}}(x) d x}{\int\left[\frac{p_{1} \theta_{1}}{x^{2}} e^{-\theta_{1} / x}+\frac{\left(1-p_{1}\right) \theta_{2}}{x^{2}} e^{-\theta_{2} / x}\right] \mu_{\widetilde{x_{l}}}(x) d x}=0
\end{aligned}
$$

The solution of the two nonlinear Likelihood equations (9) and (10) yields the MLEs of $\theta_{1}$ and $\theta_{2}$ respectively.Since there are no closed forms of the solutions, iterative approximation techniques can be used to obtain the MLEs.

In the following, we consider iterative approximation techniques namely NewtonRaphson (NR) algorithm to determine the MLEs of the parameters $\theta_{1}$ and $\theta_{2}$.

\section{Newton-Raphson (NR) Algorithm:}

In this algorithm, the solution of the Likelihood equation is obtained through an iterative procedure.

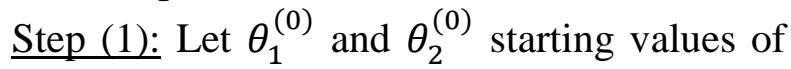
$\theta_{1}$ and $\theta_{2}$ when $h=0$.

$$
\frac{\partial^{2} \ell\left(\theta_{1}, \theta_{2} \mid \underline{\tilde{x}}\right)}{\partial \theta_{1}^{2}}=\sum_{i=1}^{n} \frac{\int\left[\frac{-2 p_{1}}{x^{3}} e^{-\theta_{1} / x}+\frac{p_{1} \theta_{1}}{x^{4}} e^{-\theta_{1} / x}\right] \mu_{\widetilde{x_{l}}}(x) d x}{\int\left[\frac{p_{1} \theta_{1}}{x^{2}} e^{-\theta_{1} / x}+\frac{\left(1-p_{1}\right) \theta_{2}}{x^{2}} e^{-\theta_{2} / x}\right] \mu_{\widetilde{x_{l}}}(x) d x}\left(\frac{\int\left[\frac{p_{1}}{x^{2}} e^{-\theta_{1} / x}-\frac{p_{1} \theta_{1}}{x^{3}} e^{-\theta_{1} / x}\right] \mu_{\widetilde{x_{l}}}(x) d x}{\int\left[\frac{p_{1} \theta_{1}}{x^{2}} e^{-\theta_{1} / x}+\frac{\left(1-p_{1}\right) \theta_{2}}{x^{2}} e^{-\theta_{2} / x}\right] \mu_{\widetilde{x_{l}}}(x) d x}\right)^{2}
$$

Step (2): At iteration $(h+1)$, estimate the new value of $\theta_{1}$ and $\theta_{2}$, as:

$$
\begin{aligned}
{\left[\begin{array}{l}
\hat{\theta}_{1}^{(h+1)} \\
\hat{\theta}_{2}^{(h+1)}
\end{array}\right]=\left[\begin{array}{l}
\hat{\theta}_{1}^{(h)} \\
\hat{\theta}_{2}^{(h)}
\end{array}\right]-} \\
{\left[\begin{array}{cc}
\frac{\partial^{2} \ell}{\partial \theta_{1}^{2}} & \frac{\partial^{2} \ell}{\partial \theta_{1} \partial \theta_{2}} \\
\frac{\partial^{2} \ell}{\partial \theta_{2} \partial \theta_{1}} & \frac{\partial^{2} \ell}{\partial \theta^{2}}
\end{array}\right]_{\theta_{1}=\widehat{\theta}_{1}^{(h)}}^{-1}\left[\begin{array}{c}
\frac{\partial \ell}{\partial \theta_{1}} \\
\frac{\partial \ell}{\partial \theta_{2}}
\end{array}\right] }
\end{aligned}
$$

where the first-order derivatives of the natural Log-Likelihood with respect to the parameters $\theta_{1}$ and $\theta_{2}$, required for proceeding with the NR-algorithm, are obtained as in the equation (9) and (10) and the second-order derivatives are obtained, as follows: 


$$
\begin{aligned}
& \frac{\partial^{2} \ell\left(\theta_{1}, \theta_{2} \mid \underline{\tilde{x}}\right)}{\partial \theta_{1} \partial \theta_{2}}=\sum_{i=1}^{n} \frac{\left(\int\left[\frac{p_{1}}{x^{2}} e^{-\theta_{1} / x}-\frac{p_{1} \theta_{1}}{x^{3}} e^{-\theta_{1} / x}\right] \mu_{\widetilde{x_{l}}}(x) d x\right)}{\left(\int\left[\frac{p_{1} \theta_{1}}{x^{2}} e^{-\theta_{1} / x}+\frac{\left(1-p_{1}\right) \theta_{2}}{x^{2}} e^{-\theta_{2} / x}\right] \mu_{\widetilde{x_{l}}}(x) d x\right)^{2}} \times \\
& \left(\int\left[\frac{1-p_{1}}{x^{2}} e^{-\theta_{2} / x}-\frac{\left(1-p_{1}\right) \theta_{2}}{x^{3}} e^{-\theta_{2} / x}\right] \mu_{\widetilde{x_{l}}}(x) d x\right) \\
& \frac{\partial^{2} \ell\left(\theta_{1}, \theta_{2} \mid \underline{\tilde{x}}\right)}{\partial \theta_{2}^{2}}=\sum_{i=1}^{n} \frac{\left(\int\left[\frac{-2\left(1-p_{1}\right)}{x^{3}} e^{-\theta_{2} / x}+\frac{\left(1-p_{1}\right) \theta_{2}}{x^{4}} e^{-\theta_{2} / x}\right] \mu_{\widetilde{x}_{l}}(x) d x\right)}{\left(\int\left[\frac{p_{1} \theta_{1}}{x^{2}} e^{-\theta_{1} / x}+\frac{\left(1-p_{1}\right) \theta_{2}}{x^{2}} e^{-\theta_{2} / x}\right] \mu_{\widetilde{x_{l}}}(x) d x\right)} \\
& (-)\left(\frac{\int\left[\frac{\left(1-p_{1}\right)}{x^{2}} e^{-\theta_{2} / x}-\frac{\left(1-p_{1}\right) \theta_{2}}{x^{3}} e^{-\theta_{2} / x}\right] \mu_{\widetilde{x_{l}}}(x) d x}{\left(\int\left[\frac{p_{1} \theta_{1}}{x^{2}} e^{-\theta_{1} / x}+\frac{\left(1-p_{1}\right) \theta_{2}}{x^{2}} e^{-\theta_{2} / x}\right] \mu_{\widetilde{x_{l}}}(x) d x\right)}\right)^{2}
\end{aligned}
$$

Step (3): Repeat step (2) until convergence occurs, i.e., $\left|\hat{\theta}_{1}^{(h+1)}-\hat{\theta}_{1}^{(h)}\right|+\mid \hat{\theta}_{2}^{(h+1)}-$ $\hat{\theta}_{2}^{(h)} \mid<\varepsilon$, for some pre-fixed $\varepsilon>0$. When the convergence occurs then the current $\hat{\theta}_{1}^{(h+1)}$ and $\hat{\theta}_{2}^{(h+1)}$ represent the maximum Likelihood estimate of $\theta_{1}$ and $\theta_{2}$ via NR algorithm which we referred to as $\left(\hat{\theta}_{1 M L}^{N R}, \hat{\theta}_{2 M L}^{N R}\right)$.

In the following, we provide Bayesian estimations of the parameters of MTIED when the available data are in the form of fuzzy numbers.

\section{Bayes Estimations of the Parameters}

For a Bayesian estimation of the unknown parameters, we need prior distributions for these parameters. Consider the prior distributions of $\theta_{1}$ and $\theta_{2}$ of MTIED are taken to be independent $\operatorname{Gamma}(a, b)$ and Gamma $(c, d)$ respectively with p.d.fs.

$$
\begin{aligned}
& \pi_{1}\left(\theta_{1}\right)=\frac{b^{a}}{\Gamma(a)} \theta_{1}^{a-1} e^{-b \theta_{1}} ; \theta_{1}>0, a, b>0 \\
& \pi_{2}\left(\theta_{2}\right)=\frac{d^{c}}{\Gamma(c)} \theta_{2}^{c-1} e^{-d \theta_{2}} ; \theta_{2}>0, c, d>0
\end{aligned}
$$

leads to a joint prior distribution of $\theta_{1}$ and $\theta_{2}$ of the form:

$$
\begin{aligned}
\pi\left(\theta_{1}, \theta_{2}\right) & =\pi_{1}\left(\theta_{1}\right) \cdot \pi_{2}\left(\theta_{2}\right) \\
& =\frac{b^{a} d^{c}}{\Gamma(a) \Gamma(c)} \theta_{1}^{(a-1)} \theta_{2}^{(c-1)} e^{-\left(b \theta_{1}+d \theta_{2}\right)}
\end{aligned}
$$

The joint posterior density function of $\theta_{1}$ and $\theta_{2}$ given fuzzy data can be obtained by combining (7) and (17)

$\Pi\left(\theta_{1}, \theta_{2} \mid \underline{\tilde{x}}\right)=\frac{\pi\left(\theta_{1}, \theta_{2} \mid \underline{\tilde{x}}\right)}{\int_{\theta_{2}} \int_{\theta_{1}} \pi\left(\theta_{1}, \theta_{2} \mid \underline{\tilde{x}}\right) d \theta_{1} d \theta_{2}}$

where:

$\pi\left(\theta_{1}, \theta_{2} \mid \underline{\tilde{x}}\right)=L\left(\theta_{1}, \theta_{2} \mid \underline{\tilde{x}}\right) \pi\left(\theta_{1}, \theta_{2}\right)$

$$
\begin{aligned}
= & \frac{b^{a} d^{c}}{\Gamma(a) \Gamma(c)} \theta_{1}^{(a-1)} \theta_{2}^{(c-1)} e^{-\left(b \theta_{1}+d \theta_{2}\right)} \times \\
& \prod_{i=1}^{n} \int\left[\frac{p_{1} \theta_{1}}{x^{2}} e^{-\theta_{1} / x}+(1-\right. \\
& \left.\left.p_{1}\right) \frac{\theta_{2}}{x^{2}} e^{-\theta_{2} / x}\right] \mu_{\widetilde{x_{l}}}(x) d x
\end{aligned}
$$

The squared error loss function (SELF) was proposed by Legendre (1805) and Gauss (1810) in order to develop least square theory. The formula of this loss function for $\theta$ is, [1] $L(\theta, \hat{\theta})=(\hat{\theta} \theta)^{2}$ according to eq.(19), Bayes estimator of $\theta$ based on SELF is obtained by:

$\hat{\theta}=E(\theta \mid \underline{\tilde{x}})$

So, Bayes estimation of any function of the parameters, say $w\left(\theta_{1}, \theta_{2}\right)$, under a squared error loss function, $\widehat{w}_{B S}\left(\theta_{1}, \theta_{2}\right)$, can be written as:

$$
\begin{gathered}
\left.\widehat{w}_{B S}\left(\theta_{1}, \theta_{2}\right)=E\left(w\left(\theta_{1}, \theta_{2}\right) \mid \underline{\tilde{x}}\right)\right)= \\
\frac{\int_{0}^{\infty} \int_{0}^{\infty} w\left(\theta_{1}, \theta_{2}\right) \pi\left(\theta_{1}, \theta_{2} \mid \underline{\tilde{x}}\right) d \theta_{1} d \theta_{2}}{\int_{0}^{\infty} \int_{0}^{\infty} \pi\left(\theta_{1}, \theta_{2} \mid \underline{\tilde{x}}\right) d \theta_{1} d \theta_{2}}
\end{gathered}
$$

Note that, Bayes estimators in (21) is of the form of ratio of two integrals, which cannot be simplified in to a closed form. However, we can approximate this Bayes estimator into a form containing no integrals by using the Lindley's approximation form.

\section{Lindley's Approximation:}

$\left.E\left(w\left(\theta_{1}, \theta_{2}\right) \mid \underline{\tilde{x}}\right)\right)$

$=\frac{\int_{0}^{\infty} \int_{0}^{\infty} w\left(\theta_{1}, \theta_{2}\right) e^{\left.\ell\left(\theta_{1}, \theta_{2}\right) \mid \underline{\tilde{x}}\right)+\rho\left(\theta_{1}, \theta_{2}\right)} d \theta_{1} d \theta_{2}}{\int_{0}^{\infty} \int_{0}^{\infty} e^{\left.\ell\left(\theta_{1}, \theta_{2}\right) \mid \underline{\tilde{x}}\right)+\rho\left(\theta_{1}, \theta_{2}\right)} d \theta_{1} d \theta_{2}}$

where:

$w\left(\theta_{1}, \theta_{2}\right)$ is a function of $\theta_{1}$ and $\theta_{2}$ only, $\ell\left(\theta_{1}, \theta_{2} \mid \underline{\tilde{x}}\right)$ is natural Log-Likelihood function defined by $(8), \rho\left(\theta_{1}, \theta_{2}\right)$ is natural Log-joint prior density function. 
Now, according to Lindley, for sufficiently Large sample size, the ratio of integral $\left.I(\underline{\tilde{x}})=E\left(w\left(\theta_{1}, \theta_{2}\right) \mid \underline{\tilde{x}}\right)\right)$ appears in equation (22) can be written as, (see [2], [9])

$$
\begin{aligned}
& I(\underline{\tilde{x}})=\widehat{w}+\frac{1}{2}\left[\left(\widehat{w}_{\theta_{2} \theta_{2}}+2 \widehat{w}_{\theta_{2}} \hat{\rho}_{\theta_{2}}\right) \hat{\sigma}_{\theta_{2} \theta_{2}}+\right. \\
& \left(\widehat{w}_{\theta_{2} \theta_{1}}+2 \widehat{w}_{\theta_{2}} \hat{\rho}_{\theta_{1}}\right) \hat{\sigma}_{\theta_{2} \theta_{1}}+\left(\widehat{w}_{\theta_{1} \theta_{2}}+\right. \\
& \left.2 \widehat{w}_{\theta_{1}} \hat{\rho}_{\theta_{2}}\right) \hat{\sigma}_{\theta_{1} \theta_{2}}+ \\
& \left.\left(\widehat{w}_{\theta_{1} \theta_{1}}+2 \widehat{w}_{\theta_{1}} \hat{\rho}_{\theta_{1}}\right) \hat{\sigma}_{\theta_{1} \theta_{1}}\right]+ \\
& \frac{1}{2}\left[\left(\widehat{w}_{\theta_{1}} \hat{\sigma}_{\theta_{2} \theta_{1}}+\right.\right. \\
& \left.\widehat{w}_{\theta_{2}} \hat{\sigma}_{\theta_{2} \theta_{2}}\right)\left(\hat{\ell}_{\theta_{2} \theta_{1} \theta_{1}} \hat{\sigma}_{\theta_{1} \theta_{1}}+\right. \\
& \hat{\ell}_{\theta_{1} \theta_{2} \theta_{2}} \hat{\sigma}_{\theta_{1} \theta_{2}}+\hat{\ell}_{\theta_{2} \theta_{1} \theta_{2}} \hat{\sigma}_{\theta_{2} \theta_{1}}+ \\
& \left.\hat{\ell}_{\theta_{2} \theta_{2} \theta_{2}} \hat{\sigma}_{\theta_{2} \theta_{2}}\right)+\left(\widehat{w}_{\theta_{1}} \hat{\sigma}_{\theta_{1} \theta_{1}}+\right. \\
& \left.\widehat{w}_{\theta_{2}} \hat{\sigma}_{\theta_{1} \theta_{2}}\right)\left(\hat{\ell}_{\theta_{1} \theta_{1} \theta_{1}} \hat{\sigma}_{\theta_{1} \theta_{1}}+\right. \\
& \hat{\ell}_{\theta_{1} \theta_{2} \theta_{1}} \hat{\sigma}_{\theta_{1} \theta_{2}}+\hat{\ell}_{\theta_{2} \theta_{1} \theta_{1}} \hat{\sigma}_{\theta_{2} \theta_{1}}+ \\
& \left.\left.\hat{\ell}_{\theta_{2} \theta_{2} \theta_{1}} \hat{\sigma}_{\theta_{2} \theta_{2}}\right)\right]
\end{aligned}
$$

where $\hat{\theta}_{1}$ and $\hat{\theta}_{2}$ are the MLE's of $\theta_{1}$ and $\theta_{2}$ respectively, $\sigma_{i j}$ is the $(i, j)^{\text {th }}$ elements of matrix $\quad\left[(-) \frac{\partial^{2} \ell\left(\theta_{1}, \theta_{2} \mid \underline{\tilde{x}}\right)}{\partial \theta_{1} \partial \theta_{2}}\right]^{-1} ; i, j=1,2$. Subscripts $(i, j)$ refer to $\theta_{1}, \theta_{2}$ respectively, and:

$\widehat{w}_{\theta_{1}}=\left.\frac{\partial w}{\partial \theta_{1}}\right|_{\begin{array}{c}\theta_{1}=\widehat{\theta}_{1} \\ \theta_{2}=\widehat{\theta}_{2}\end{array}} ; \widehat{w}_{\theta_{1} \theta_{2}}=\left.\frac{\partial^{2} w}{\partial \theta_{1} \partial \theta_{2}}\right|_{\begin{array}{c}\theta_{1}=\widehat{\theta}_{1} \\ \theta_{2}=\widehat{\theta}_{2}\end{array}} ;$

$\widehat{w}_{\theta_{1} \theta_{1}}=\left.\frac{\partial^{2} w}{\partial \theta_{1}{ }^{2}}\right|_{\theta_{1}=\widehat{\theta}_{1}} ; \widehat{w}_{\theta_{2}}=\left.\frac{\partial w}{\partial \theta_{2}}\right|_{\theta_{1}=\widehat{\theta}_{1}} ;$ $\theta_{2}=\widehat{\theta}_{2} \quad \theta_{2}=\widehat{\theta}_{2}$

$\widehat{w}_{\theta_{2} \theta_{1}}=\left.\frac{\partial^{2} w}{\partial \theta_{2} \partial \theta_{1}}\right|_{\theta_{1}=\widehat{\theta}_{1}} ; \widehat{w}_{\theta_{2} \theta_{2}}=\left.\frac{\partial^{2} w}{\partial \theta_{2}{ }^{2}}\right|_{\theta_{1}=\widehat{\theta}_{1}}$

$\hat{\rho}_{\theta_{1}}=\left.\frac{\partial \ln \pi\left(\theta_{1}, \theta_{2}\right)}{\partial \theta_{1}}\right|_{\theta_{1}=\widehat{\theta}_{1}} ^{\theta_{2}=\widehat{\theta}_{2}}=\frac{a-1}{\theta_{1}}-b ;$

$\theta_{2}=\widehat{\theta}_{2}$

$$
\begin{aligned}
& \hat{\rho}_{\theta_{1}}=\left.\frac{\partial \ln \pi\left(\theta_{1} \theta_{2}\right)}{\partial \theta_{1}}\right|_{\begin{array}{c}
\theta_{1}=\widehat{\theta}_{1} \\
\theta_{2}=\widehat{\theta}_{2}
\end{array}}=\frac{a-1}{\theta_{1}}-b ; \\
& \hat{\rho}_{\theta_{2}}=\left.\frac{\partial \ln \pi\left(\theta_{1}, \theta_{2}\right)}{\partial \theta_{2}}\right|_{\theta_{1}=\widehat{\theta}_{1}}=\frac{c-1}{\theta_{2}}-d ;
\end{aligned}
$$

Now:

$$
\begin{aligned}
& \hat{\ell}_{\theta_{1} \theta_{1}}=\left.\frac{\partial^{2} \ell\left(\theta_{1}, \theta_{2} \mid \underline{\tilde{x}}\right)}{\partial \theta_{1}^{2}}\right|_{\substack{\theta_{1}=\widehat{\theta}_{1} \\
\theta_{2}=\widehat{\theta}_{2}}} \text { as in (12) } \\
& \hat{\ell}_{\theta_{1} \theta_{2}}=\left.\frac{\partial^{2} \ell\left(\theta_{1}, \theta_{2} \mid \underline{\tilde{x}}\right)}{\partial \theta_{1} \partial \theta_{2}}\right|_{\theta_{1}=\widehat{\theta}_{1}}=\hat{\ell}_{\theta_{2} \theta_{1}}= \\
& \left.\frac{\partial^{2} \ell\left(\theta_{1}, \theta_{2} \mid \underline{\tilde{x}}\right)}{\partial \theta_{2} \partial \theta_{1}}\right|_{\theta_{1}=\widehat{\theta}_{1}} ^{\theta_{2}=\widehat{\theta}_{2}} \text { as in (13) } \\
& \hat{\ell}_{\theta_{2} \theta_{2}}=\left.\frac{\partial^{2} \ell\left(\theta_{1}, \theta_{2} \mid \underline{\tilde{x}}\right)}{\partial \theta_{2}^{2}}\right|_{\theta_{1}=\widehat{\theta}_{1}} \text { as in (14) } \\
& \hat{\ell}_{\theta_{1} \theta_{1} \theta_{1}}=\left.\frac{\partial^{3} \ell\left(\theta_{1}, \theta_{2} \mid \underline{\tilde{x}}\right)}{\partial \theta_{1}^{3}}\right|_{\theta_{1}=\widehat{\theta}_{1}} \\
& \theta_{2}=\widehat{\theta}_{2}
\end{aligned}
$$

$$
\begin{aligned}
& =\sum_{i=1}^{n} \frac{\int\left[\frac{3 p_{1}}{x^{4}} e^{-\theta_{1} / x}-\frac{\theta_{1} p_{1}}{x^{5}} e^{-\theta_{1} / x}\right] \mu_{\widetilde{x_{l}}}(x) d x}{\int\left[\frac{p_{1} \theta_{1}}{x^{2}} e^{-\theta_{1} / x}+\frac{\left(1-p_{1}\right) \theta_{2}}{x^{2}} e^{-\theta_{2} / x}\right] \mu_{\widetilde{x_{l}}}(x) d x} \\
& (-3) \sum_{i=1}^{n} \frac{\left(\int\left[\frac{-2 p_{1}}{x^{3}} e^{-\theta_{1} / x}+\frac{p_{1} \theta_{1}}{x^{4}} e^{-\theta_{1} / x}\right] \mu_{\widetilde{x}_{l}}(x) d x\right)}{\left(\int\left[\frac{p_{1} \theta_{1}}{x^{2}} e^{-\theta_{1} / x}+\frac{\left(1-p_{1}\right) \theta_{2}}{x^{2}} e^{-\theta_{2} / x}\right] \mu_{{\widetilde{x_{l}}}_{1}}(x) d x\right)^{2}} \times \\
& \left(\int\left[\frac{p_{1}}{x^{2}} e^{-\theta_{1} / x}-\frac{p_{1} \theta_{1}}{x^{3}} e^{-\theta_{1} / x}\right] \mu_{\widetilde{x}_{l}}(x) d x\right)(+2) \\
& \sum_{i=1}^{n}\left(\frac{\left(\int\left[\frac{p_{1}}{x^{2}} e^{-\theta_{1} / x}-\frac{p_{1} \theta_{1}}{x^{3}} e^{-\theta_{1} / x}\right] \mu_{\widetilde{x}_{l}}(x) d x\right)}{\left(\int\left[\frac{p_{1} \theta_{1}}{x^{2}} e^{-\theta_{1} / x}+\frac{\left(1-p_{1}\right) \theta_{2}}{x^{2}} e^{-\theta_{2} / x}\right] \mu_{\widetilde{x_{l}}}(x) d x\right)}\right)^{3} \\
& \hat{\ell}_{\theta_{2} \theta_{2} \theta_{2}}=\left.\frac{\partial^{3} \ell\left(\theta_{1}, \theta_{2} \mid \tilde{\underline{x}}\right)}{\partial \theta_{2}^{3}}\right|_{\begin{array}{r}
\theta_{1}=\widehat{\theta}_{1} \\
\theta_{2}=\widehat{\theta}_{2}
\end{array}} \\
& =\sum_{i=1}^{n} \frac{\int\left[\frac{3\left(1-p_{1}\right)}{x^{4}} e^{-\theta_{2} / x}-\frac{\left(1-p_{1}\right) \theta_{2}}{x^{5}} e^{-\theta_{2} / x}\right] \mu_{\widetilde{x_{l}}}(x) d x}{\int\left[\frac{p_{1} \theta_{1}}{x^{2}} e^{-\theta_{1} / x}+\frac{\left(1-p_{1}\right) \theta_{2}}{x^{2}} e^{-\theta_{2} / x}\right] \mu_{\widetilde{x_{l}}}(x) d x}- \\
& 3 \sum_{i=1}^{n} \frac{\left(\int\left[\frac{-2\left(1-p_{1}\right)}{x^{3}} e^{-\theta_{2} / x}+\frac{\left(1-p_{1}\right) \theta_{2}}{x^{4}} e^{-\theta_{2} / x}\right] \mu_{{\widetilde{x_{l}}}_{l}}(x) d x\right)}{\left(\int\left[\frac{p_{1} \theta_{1}}{x^{2}} e^{-\theta_{1} / x}+\frac{\left(1-p_{1}\right) \theta_{2}}{x^{2}} e^{-\theta_{2} / x}\right] \mu_{{\widetilde{x_{l}}}_{l}}(x) d x\right)^{2}} \times \\
& \left(\int\left[\frac{\left(1-p_{1}\right)}{x^{2}} e^{-\theta_{2} / x}-\frac{\left(1-p_{1}\right) \theta_{2}}{x^{3}} e^{-\theta_{2} / x}\right] \mu_{\widetilde{x}_{l}}(x) d x\right) \\
& +2 \sum_{i=1}^{n}\left(\frac{\left(\int\left[\frac{\left(1-p_{1}\right)}{x^{2}} e^{-\theta_{2} / x}-\frac{\left(1-p_{1}\right) \theta_{2}}{x^{3}} e^{-\theta_{2} / x}\right] \mu_{\widetilde{x}_{\underline{l}}}(x) d x\right)}{\left(\int\left[\frac{p_{1} \theta_{1}}{x^{2}} e^{-\theta_{1} / x_{+}}+\frac{\left(1-p_{1}\right) \theta_{2}}{x^{2}} e^{-\theta_{2} / x}\right] \mu_{\widetilde{x_{l}}}(x) d x\right)}\right)^{3}
\end{aligned}
$$

$$
\hat{\ell}_{\theta_{2} \theta_{1} \theta_{1}}=\left.\frac{\left.\partial^{3} \ell\left(\theta_{1}, \theta_{2}\right) \mid \tilde{x}\right)}{\partial \theta_{2} \partial \theta_{1} \partial \theta_{1}}\right|_{\theta_{1}=\widehat{\theta}_{1}}
$$$$
=-\sum_{i=1}^{n} \frac{\left(\int\left[\frac{\left(1-p_{1}\right)}{x^{2}} e^{-\theta_{2} / x_{-}}-\frac{\left(1-p_{1}\right) \theta_{2}}{x^{3}} e^{-\theta_{2} / x}\right] \mu_{\widehat{x}_{l}}(x) d x\right)}{\left(\int\left[\frac{p_{1} \theta_{1}}{x^{2}} e^{-\theta_{1} / x}+\frac{\left(1-p_{1}\right) \theta_{2}}{x^{2}} e^{-\theta_{2} / x}\right] \mu_{\widetilde{x}_{l}}(x) d x\right)^{2}} \times
$$$$
\left(\int\left[\frac{p_{1} \theta_{1}}{x^{4}} e^{-\theta_{1} / x}-\frac{2 p_{1}}{x^{3}} e^{-\theta_{1} / x}\right] \mu_{\widetilde{x}_{l}}(x) d x\right)+
$$$$
2 \sum_{i=1}^{n} \frac{\left(\int\left[\frac{\left(1-p_{1}\right)}{x^{2}} e^{-\theta_{2} / x}-\frac{\left(1-p_{1}\right) \theta_{2}}{x^{3}} e^{-\theta_{2} / x}\right] \mu_{\widetilde{x}_{l}}(x) d x\right)}{\left(\int\left[\frac{p_{1} \theta_{1}}{x^{2}} e^{-\theta_{1} / x}+\frac{\left(1-p_{1}\right) \theta_{2}}{x^{2}} e^{-\theta_{2} / x}\right] \mu_{\widetilde{x_{l}}}(x) d x\right)^{3}} \times
$$$$
\left(\int\left[\frac{p_{1}}{x^{2}} e^{-\theta_{1} / x}-\frac{p_{1} \theta_{1}}{x^{3}} e^{-\theta_{1} / x}\right] \mu_{{\widetilde{x_{l}}}_{l}}(x) d x\right)^{2}
$$$$
\hat{\ell}_{\theta_{2} \theta_{1} \theta_{2}}=\left.\frac{\partial^{2} \ell\left(\theta_{1}, \theta_{2} \mid \underline{\tilde{x}}\right)}{\partial \theta_{2} \partial \theta_{1} \partial \theta_{2}}\right|_{\theta_{1}=\widehat{\theta}_{1}}
$$$$
=(-) \sum_{i=1}^{n} \frac{\left(\int\left[\frac{p_{1}}{x^{2}} e^{-\theta_{1} / x}-\frac{\theta_{2}=\hat{p}_{1} \theta_{1}}{x^{3}} e^{-\theta_{1} / x}\right] \mu_{{\widetilde{x_{l}}}_{1}}(x) d x\right)}{\left(\int\left[\frac{p_{1} \theta_{1}}{x^{2}} e^{-\theta_{1} / x}+\frac{\left(1-p_{1}\right) \theta_{2}}{x^{2}} e^{-\theta_{2} / x}\right] \mu_{\widetilde{x_{l}}}(x) d x\right)^{2}}
$$$$
\times\left(\int\left[\frac{-2\left(1-p_{1}\right)}{x^{3}} e^{-\theta_{2} / x}+\frac{\left(1-p_{1}\right) \theta_{2}}{x^{4}} e^{-\theta_{2} / x}\right] \mu_{\widetilde{x}_{l}}(x) d x\right)
$$$$
+2 \sum_{i=1}^{n} \frac{\left(\int\left[\frac{\left(1-p_{1}\right)}{x^{2}} e^{-\theta_{2} / x_{-}} \frac{\left(1-p_{1}\right) \theta_{2}}{x^{3}} e^{-\theta_{2} / x}\right] \mu_{\widetilde{x_{l}}}(x) d x\right)^{2}}{\left(\int\left[\frac{p_{1} \theta_{1}}{x^{2}} e^{-\theta_{1} / x_{+}}+\frac{\left(1-p_{1}\right) \theta_{2}}{x^{2}} e^{-\theta_{2} / x}\right] \mu_{\widetilde{x_{l}}}(x) d x\right)^{3}}
$$$$
\times\left(\int\left[\frac{p_{1}}{x^{2}} e^{-\theta_{1} / x}-\frac{p_{1} \theta_{1}}{x^{3}} e^{-\theta_{1} / x}\right] \mu_{\widetilde{x_{l}}}(x) d x\right)
$$

\subsection{Bayes estimation based on square error loss function:}

The two parameters $\theta_{1}$ and $\theta_{2}$ and reliability function can be estimate by using 
Lindely approximation from eq. (23), as follows:

Approximate Bayes Estimate of $\theta_{1}$ based on SELF:

Assume that $w$ in (23) equal to $w\left(\theta_{1}, \theta_{2}\right)=\theta_{1}$ and then:

$w_{\theta_{1}}=1, w_{\theta_{1} \theta_{1}}=w_{\theta_{2}}=w_{\theta_{2} \theta_{2}}=w_{\theta_{1} \theta_{2}}=$ $w_{\theta_{2} \theta_{1}}=0$.

Now, Bayes estimate of $\theta_{1}$ based on SELF according to Lindley's approximation referred to as $\hat{\theta}_{1_{B S}}^{L}$ can be obtained by the following expression,

$\hat{\theta}_{1_{B S}}^{L}=E\left(\theta_{1} \mid \underline{\tilde{x}}\right)$

\section{Approximate Bayes Estimate of $\theta_{2}$ based on SELF:}

Assume that $w$ in (23) equal to $w\left(\theta_{1}, \theta_{2}\right)=\theta_{2}$, and then:

$w_{\theta_{2}}=1, w_{\theta_{2} \theta_{2}}=w_{\theta_{1}}=w_{\theta_{1} \theta_{1}}=w_{\theta_{1} \theta_{2}}=$ $w_{\theta_{2} \theta_{1}}=0$.

Now, Bayes estimate of $\theta_{2}$ based on SELF according to Lindley's approximation referred to as $\hat{\theta}_{2_{B S}}^{L}$, can be obtained by the following expression:

$\hat{\theta}_{2_{B S}}^{L}=E\left(\theta_{2} \mid \underline{\tilde{x}}\right)$

Approximate Bayes Estimate of $R(t)$ based on SELF:

Assume that $w$ in (23) equal to:

$w\left(\theta_{1}, \theta_{2}\right)=\mathrm{R}(\mathrm{t})$

$$
\begin{aligned}
= & p_{1}\left(1-e^{-\theta_{1} / t}\right)+\left(1-p_{1}\right)(1- \\
& \left.e^{-\theta_{2} / t}\right)
\end{aligned}
$$

and then:

$w_{\theta_{1}}=\frac{p_{1}}{t} e^{-\theta_{1} / t}, w_{\theta_{1} \theta_{1}}=\frac{-p_{1}}{t^{2}} e^{-\theta_{1} / t}$

$w_{\theta_{2}}=\frac{\left(1-p_{1}\right)}{t} e^{-\theta_{2} / t}, w_{\theta_{2} \theta_{2}}=\frac{-\left(1-p_{1}\right)}{t^{2}} e^{-\theta_{2} / t}$,

$w_{\theta_{1} \theta_{2}}=w_{\theta_{2} \theta_{1}}=0$.

Now, Bayes estimate of $\mathrm{R}(\mathrm{t})$ based on SELF according to Lindley's approximation, referred to as $\hat{R}_{B S}^{L}(t)$, can be obtained by the following expression:

$\hat{R}_{B S}^{L}(t)=E(R(t) \mid \underline{\tilde{x}})$

\subsection{Bayes estimation based on quadratic loss function:}

De Groot (1970) [4] discussed different types of loss function and obtained the Bayes estimates based on quadratic loss function $(Q L F)$ which is defined as:

$L(\theta, \hat{\theta})=\left(1-\frac{\widehat{\theta}}{\theta}\right)$

According to eq. (31), Bayes estimator of $\theta$ based on QLF is obtained by [3]:

$\hat{\theta}_{B Q}=\frac{E\left(\frac{1}{\theta} \mid \underline{\tilde{x}}\right)}{E\left(\frac{1}{\theta^{2}} \mid \underline{\underline{x}}\right)}$

Bayes estimate of scale parameters $\theta_{1}, \theta_{2}$ and reliability function $\mathrm{R}(\mathrm{t})$ based on QLF can be obtained as follows:

Approximate Bayes Estimate of $\theta_{1}$ based on QLF:

For the estimation of the scale parameter $\theta_{1}$ of MTIED based on quadratic loss function according to Lindley's approximation referred to as $\theta_{1_{B Q}}^{L}$ can be obtained by the following expression,

$\widehat{\theta}_{1 \mathrm{BQ}}^{\mathrm{L}}=\frac{E\left(\frac{1}{\theta_{1}} \mid \underline{\tilde{x}}\right)}{E\left(\frac{1}{\theta_{1}^{2}} \mid \underline{\underline{x}}\right)}$

Put $w$ in (23) equal to:

1) $w\left(\theta_{1}, \theta_{2}\right)=\frac{1}{\theta_{1}}$, and then $w_{\theta_{1}}=\frac{-1}{\theta_{1}{ }^{2}}$, $w_{\theta_{1} \theta_{1}}=\frac{2}{\theta_{1}{ }^{3}}, \quad w_{\theta_{2}}=w_{\theta_{2} \theta_{2}}=w_{\theta_{1} \theta_{2}}=$ $w_{\theta_{2} \theta_{1}}=0$.

2) $w\left(\theta_{1}, \theta_{2}\right)=\frac{1}{\theta_{1}{ }^{2}}$, and then $w_{\theta_{1}}=\frac{-2}{\theta_{1}{ }^{3}}$, $w_{\theta_{1} \theta_{1}}=\frac{6}{\theta_{1}^{4}}, \quad w_{\theta_{2}}=w_{\theta_{2} \theta_{2}}=w_{\theta_{1} \theta_{2}}=$ $w_{\theta_{2} \theta_{1}}=0$.

\section{Approximate Bayes Estimate of $\theta_{2}$ based on QLF:}

For the estimation of the scale parameter $\theta_{2}$ of MTIED based on quadratic loss function according to Lindley's approximation referred to as $\theta_{2}^{L}{ }_{B Q}^{L}$ can be obtained by the following expression:

$\hat{\theta}_{2_{B Q}}^{L}=\frac{E\left(\frac{1}{\theta_{2}} \mid \underline{\tilde{x}}\right)}{E\left(\frac{1}{\theta_{2}{ }^{2}} \mid \underline{\tilde{x}}\right)}$

Assume that $w$ in (23) equal to:

1) $w\left(\theta_{1}, \theta_{2}\right)=\frac{1}{\theta_{2}}$, and then $w_{\theta_{2}}=\frac{-1}{\theta_{2}{ }^{2}}$, $w_{\theta_{2} \theta_{2}}=\frac{2}{\theta_{2}{ }^{3}}, \quad w_{\theta_{1}}=w_{\theta_{1} \theta_{1}}=w_{\theta_{1} \theta_{2}}=$ $w_{\theta_{2} \theta_{1}}=0$. 
2) $w\left(\theta_{1}, \theta_{2}\right)=\frac{1}{\theta_{2}{ }^{2}}$, and then $w_{\theta_{2}}=\frac{-2}{\theta_{2}{ }^{3}}$, $w_{\theta_{2} \theta_{21}}=\frac{6}{\theta_{2}^{4}}, \quad w_{\theta_{1}}=w_{\theta_{1} \theta_{1}}=w_{\theta_{1} \theta_{2}}=$ $w_{\theta_{2} \theta_{1}}=0$.

\section{Approximate Bayes Estimate of $R(t)$ based on $Q L F$ :}

For the estimation of $\mathrm{R}(\mathrm{t})$ of MTIED based on quadratic loss function according to Lindley's approximation referred to as $\mathrm{R}(\mathrm{t})_{B Q}^{L}$ can be obtained by the following expression,

$\hat{R}(t)_{B Q}^{L}=\frac{E\left(\frac{1}{\mathrm{R}(\mathrm{t})} \mid \underline{\tilde{x}}\right)}{E\left(\frac{1}{(\mathrm{R}(\mathrm{t}))^{2}} \mid \underline{\tilde{x}}\right)}$

Assume that $w$ in (23) equal to:

1) $w\left(\theta_{1}, \theta_{2}\right)=\frac{1}{\mathrm{R}(\mathrm{t})}$, and then

$$
\begin{aligned}
& w_{\theta_{1}}=\frac{\frac{-p_{1}}{t} e^{-\theta_{1} / t}}{(\mathrm{R}(\mathrm{t}))^{2}}, \\
& w_{\theta_{1} \theta_{1}}=\frac{\frac{p_{1}}{t^{2}} e^{-\theta_{1} / t}}{(\mathrm{R}(\mathrm{t}))^{2}}\left[1+\frac{2 p_{1} e^{-\theta_{1} / t}}{\mathrm{R}(\mathrm{t})}\right], \\
& w_{\theta_{1} \theta_{2}}=\frac{\frac{2 p_{1}\left(1-p_{1}\right)}{t^{2}} e^{-\left(\theta_{1}+\theta_{2}\right) / t}}{(\mathrm{R}(\mathrm{t}))^{3}}, \\
& w_{\theta_{2}}=\frac{\frac{-\left(1-p_{1}\right)}{t} e^{-\theta_{2} / t}}{(\mathrm{R}(\mathrm{t}))^{2}}, \\
& w_{\theta_{2} \theta_{2}}=\frac{\frac{\left(1-p_{1}\right)}{t^{2}} e^{-\theta_{2} / t}}{(\mathrm{R}(\mathrm{t}))^{2}}\left[1+\frac{2\left(1-p_{1}\right) e^{-\theta_{2} / t}}{\mathrm{R}(\mathrm{t})}\right], \\
& w_{\theta_{1} \theta_{2}}=w_{\theta_{2} \theta_{1}}=0 .
\end{aligned}
$$

2) $w\left(\theta_{1}, \theta_{2}\right)=\frac{1}{(\mathrm{R}(\mathrm{t}))^{2}}$, and then:

$$
w_{\theta_{1}}=\frac{\frac{-2 p_{1}}{t} e^{-\theta_{1} / t}}{(\mathrm{R}(\mathrm{t}))^{3}}
$$$$
w_{\theta_{1} \theta_{1}}=\frac{\frac{2 p_{1}}{t^{2}} e^{-\theta_{1} / t}}{(\mathrm{R}(\mathrm{t}))^{3}}\left[1+\frac{3 p_{1} e^{-\theta_{1} / t}}{\mathrm{R}(\mathrm{t})}\right]
$$

$$
\begin{aligned}
& w_{\theta_{2}}=\frac{\frac{-2\left(1-p_{1}\right)}{t} e^{-\theta_{2} / t}}{(\mathrm{R}(\mathrm{t}))^{3}}, \\
& w_{\theta_{2} \theta_{2}}=\frac{\frac{2\left(1-p_{1}\right)}{t^{2}} e^{-\theta_{2} / t}}{(\mathrm{R}(\mathrm{t}))^{3}}\left[1+\frac{3\left(1-p_{1}\right) e^{-\theta_{2} / t}}{\mathrm{R}(\mathrm{t})}\right], \\
& w_{\theta_{1} \theta_{2}}=\frac{\frac{6 p_{1}\left(1-p_{1}\right)}{t^{2}} e^{-\left(\theta_{1}+\theta_{2}\right) / t}}{(\mathrm{R}(\mathrm{t}))^{4}}, \\
& w_{\theta_{1} \theta_{2}}=w_{\theta_{2} \theta_{1}}=0 .
\end{aligned}
$$

\section{Simulation Study and Results}

We obtained, in the above section, MLEs and Bayesian estimates of two parameters $\theta_{1}$, $\theta_{2}$ and reliability function $\mathrm{R}(\mathrm{t})$ for mixture of MTIED. We can obtain Bayes estimation based on square error loss function (LS-NR) and quadratic loss functions (LQ-NR).The MLEs are obtained as well via the NewtonRaphson (NR) algorithm. The following algorithm will be used to generate the samples, each observation of the generated samples was made to be fuzzy observation and then calculate the estimators:

1. We have generate (100) i.i.d. random samples from the (MTIED) with different sample sizes $n_{1}=15,30,75$ for first subpopulation and $n_{2}=15,30,75$ for second subpopulation where $n=n_{1}+n_{2}$, i.e., $\quad(n=30,60,150)$ represent small, median and large sample sizes respectively, through the adoption of inverse transformation method with scale parameters $\theta_{1}=0.5,1$ for first subpopulation and $\theta_{2}=0.5,0.6$ for second subpopulation and $p=\frac{n_{1}}{n}$.

Table (1)

Parameter choices for mixture two inverse exponential distributions

\begin{tabular}{|c|c|c|}
\hline \hline Case & $\boldsymbol{\theta}_{\mathbf{1}}$ & $\boldsymbol{\theta}_{\mathbf{2}}$ \\
\hline \hline 1 & 0.5 & 0.5 \\
\hline 2 & 0.5 & 0.6 \\
\hline 3 & 1 & 0.5 \\
\hline 4 & 1 & 0.6 \\
\hline
\end{tabular}

2. Then, by employing fuzzy information system $\left\{\tilde{x}_{1}, \tilde{x}_{2}, \tilde{x}_{3}, \tilde{x}_{4}\right\}$ corresponding to the following membership functions that shown in figure (1), each observation of the generated samples was made to be fuzzy observation. 


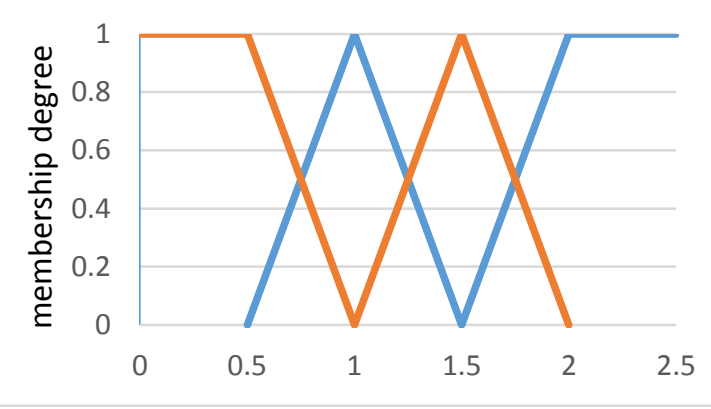

FIS used to encode the Simulated Data.

$\mu_{\check{x}_{1}}(x)=\left\{\begin{array}{c}1, x \leq 0.5 \\ \frac{1-x}{0.5}, 0.5 \leq x \leq 1 \\ 0, o . w\end{array}\right.$

$\mu_{\breve{x}_{2}}(x)=\left\{\begin{array}{c}\frac{x-0.5}{0.5}, 0.5 \leq x \leq 1 \\ \frac{1.5-x}{0.5}, 1 \leq x \leq 1.5 \\ 0, \text { o.w }\end{array}\right.$

$\mu_{\breve{x}_{3}}(x)=\left\{\begin{array}{c}\frac{x-1}{0.5}, 1 \leq x \leq 1.5 \\ \frac{1-x}{0.5}, 1.5 \leq x \leq 2 \\ 0, \text { o.w }\end{array}\right.$

$\mu_{\check{x}_{4}}(x)=\left\{\begin{array}{c}\frac{x-1.5}{0.5}, 1.5 \leq x \leq 2 \\ 1, x \geq 2 \\ 0, o . w\end{array}\right.$

3. In order to deal with non-informative gamma priors, the hyper parameters are chosen to be respectively, $a=b=c=$ $d=0.0000001$.

4. The initial values which required for proceeding algorithm are used to be symmetrical rank regression estimators. The iterative process stops when the absolute difference between two successive iteration becomes less than $\epsilon=$ 0.0001 .

5. The obtained Bayes estimates of the parameters $\theta_{1}$ and $\theta_{2}$ were compared based on average values from Mean Square Error (MSE) whereas the obtained Bayes estimates of the reliability function were compared based on average values from Integrated Mean Square Error (IMSE), where:

$$
\begin{gathered}
\operatorname{MSE}\left(\hat{\theta}_{1}\right)=\frac{\sum_{j=1}^{L}\left(\widehat{\theta}_{1}-\theta_{1}\right)^{2}}{L} \\
\operatorname{MSE}\left(\hat{\theta}_{2}\right)=\frac{\sum_{j=1}^{L}\left(\widehat{\theta}_{2}-\theta_{2}\right)^{2}}{L} \\
\operatorname{IMSE}(\hat{R}(t))=\frac{1}{L} \sum_{j=1}^{L}\left(\frac{1}{n_{t}} \sum_{i=1}^{n_{t}} \hat{R}_{j}\left(t_{i}\right)-\right. \\
\left.R\left(t_{i}\right)\right)
\end{gathered}
$$

6. The simulation program has been written by using MATLAB (R2010b) program and the computational results have been summarized in the tables (2)...(4).

\section{Conclusions and Recommendations}

- Results in tables $(2,3,4)$ appears that the MSE and IMSE values are decreasing as the sample sizes increasing.

- Tables $(2,3)$ indicate that the MLE based on NR algorithm introduced the best perform "smallest MSE values "comparing with the Bayes estimates under square error loss function(LS-NR) and the Bayes estimates under quadratic loss functions (LQ-NR) for (MTIED) with all sample sizes and four different cases.

- Table (2) increase the value of the scale parameter for first subpopulation from $\theta_{1}=0.5$ to $\theta_{1}=\mathbf{1}$, increasing the values MSE.

- Table (3) increase the value of the scale parameter for second subpopulation, from $\boldsymbol{\theta}_{\mathbf{2}}=\mathbf{0 . 5}$ to $\boldsymbol{\theta}_{\mathbf{2}}=\mathbf{0 . 6}$, increasing the values MSE associated with MLE based on NR algorithm and LS-NR.

- Table(4) indicate that the IMLE based on NR algorithm introduced the best perform comparing with LS-NR and LQ-NR for small sample size As well as for moderate sample size for all cases expect $\boldsymbol{\theta}_{\mathbf{1}}=\mathbf{1}, \boldsymbol{\theta}_{\mathbf{2}}=\mathbf{0 . 5}$

Based on conclusions stated above, for estimating the parameters of (MTIED), we recommend choosing the MLE based on NR algorithm for estimating the parameters whereas for estimating the reliability function choosing the LS-NR for large sample sizes.

\section{References}

[1] Ali, S.; Aslam, M.; Abbas, N. and Kazmi, S. (2012), Scale Parameter Estimation of the Laplace Model Using Different Asymmetric Loss Functions, International Journal of Statistics and Probability, Vol. 1, No.1, PP.105-127.

[2] Aljuaid, A. (2013), Estimating the parameters of an Exponentiated inverted Weibull Distribution under type-II censoring, Applied Mathematical Statistics, Vol. 7, No. 35, PP. 1721-1736. 
[3] Al-Noor. N. H. and Rasheed, H. Abdullah(2012), "Minimax Estimation of the Scale Parameter of the Laplace Distribution under Quadratic Loss Function", International Journal for Sciences and Technology, Vol.(7), No(3), pp. 100-107.

[4] De Geoot, M.H.(1970), Optimal Statistical Decisions McGraw-Hill Inc., United states of America.

[5] Elsherpieny, E.A., (2007), "Estimation of parameters of mixed generalized exponentially distributions from censored type I samples", Journal of applied sciences Research, Vol.3, No.12, pp.1696-1700.

[6] Everitt, B. S. and D. J. Hand, (1981)," Finite Mixture Distributions", Chapman and Hall, London.

[7] Jaheen, Z. F., (2005), "On record statistics from a mixture of two exponential distributions", Journal of Statistical Computation and Simulation, Vol.75, No.1, pp.1-11.

[8] Khan, M.S. (2012), "L-Moment and Inverse Moment Estimation of the Inverse Generalized Exponential
Distribution", International Journal of Information and Electronics Engineering, Vol.2, No.1, pp.78-82.

[9] Lindley, D. V. (1980), "Approximate Bayesian method". Trabajosa de Estadistica, 31, 223-245.

[10]Nasser, M.M. and Mahmoud, M.R. (1985), "On characterizations of a mixture of Exponential Distribution", IEEE, Vol. R-34, No.5, pp.484-488.

[11]Rider, P.R., (1961). "The method of moments applied to a mixture of two exponential distributions". Ann. Math. Statist., Vol. 32, pp.143-147.

[12] Sultan, K. S., M. A. Ismail and A. S. AlMoisheer, (2007), "Mixture of two inverse Weibull distributions: properties and estimation". Computational Statistics and Data Analysis, Computational Statistics and Data Analysis, Vol.51, pp 5377-5387.

[13]Zadeh, L. A.(1965), "Fuzzy Sets", Information and Control, 8(3): 338-353.

[14]Zadeh, L. A. (1968), "Probability Measures of Fuzzy Events", Journal of Mathematical Analysis and Applications, Vol.23, No.2, pp.421-427.

Table (2)

MSE values of the estimates of $\theta_{1}$ for different samplesizes.

\begin{tabular}{||c|c|c|c|c||}
\hline \multirow{2}{*}{ Case } & Estimate & $\widehat{\boldsymbol{\theta}}_{\mathbf{1} \boldsymbol{M} \boldsymbol{L}}^{\boldsymbol{N R}}$ & $\widehat{\boldsymbol{\theta}}_{\mathbf{1}_{\boldsymbol{B} \boldsymbol{S}} \boldsymbol{L}}$ & $\widehat{\boldsymbol{\theta}}_{\mathbf{1}_{\boldsymbol{B} \boldsymbol{Q}} \boldsymbol{L}}$ \\
\hline \hline \multirow{3}{*}{1} & Sample sizes & 0.0233932 & 0.2005009 & 0.0991962 \\
\cline { 2 - 5 } & 30 & 0.0183723 & 0.0806426 & 0.0578716 \\
\cline { 2 - 5 } & 60 & 0.0163274 & 0.0294155 & 0.0404439 \\
\hline \multirow{3}{*}{2} & 150 & 0.0158582 & 0.0459260 & 0.1170193 \\
\cline { 2 - 5 } & 30 & 0.0121490 & 0.0240735 & 0.0818985 \\
\cline { 2 - 5 } & 60 & 0.0106996 & 0.0184793 & 0.0289910 \\
\hline \multirow{3}{*}{3} & 150 & 0.2466995 & 0.6436168 & 0.6857820 \\
\cline { 2 - 5 } & 30 & 0.2360822 & 0.4862397 & 0.6218401 \\
\cline { 2 - 5 } & 60 & 0.2213613 & 0.4511455 & 0.5171946 \\
\hline \multirow{3}{*}{4} & 150 & 0.2102429 & 0.9497045 & 0.5782250 \\
\cline { 2 - 5 } & 30 & 0.2082938 & 0.5096119 & 0.5412154 \\
\cline { 2 - 5 } & 60 & 0.2043110 & 0.3965683 & 0.4589043 \\
\hline \hline
\end{tabular}


Table (3)

MSE values of the estimates of $\theta_{2}$ for different sample sizes.

\begin{tabular}{|c|c|c|c|c|}
\hline Case & Sample sizes & $\widehat{\boldsymbol{\theta}}_{2 M L}^{N R}$ & $\widehat{\boldsymbol{\theta}}_{\mathbf{2}_{B S}}^{L}$ & $\widehat{\theta}_{2_{B Q}}^{L}$ \\
\hline \multirow{3}{*}{1} & 30 & 0.0244574 & 0.1348539 & 0.0908540 \\
\hline & 60 & 0.0183518 & 0.0808005 & 0.0598297 \\
\hline & 150 & 0.0163358 & 0.0295862 & 0.0403106 \\
\hline \multirow{3}{*}{2} & 30 & 0.0457090 & 0.0881518 & 0.1482921 \\
\hline & 60 & 0.0421992 & 0.0756958 & 0.1177494 \\
\hline & 150 & 0.0393457 & 0.0730716 & 0.0938337 \\
\hline \multirow{3}{*}{3} & 30 & 0.0178490 & 0.2143132 & 0.1053265 \\
\hline & 60 & 0.0083644 & 0.0280595 & 0.0602245 \\
\hline & 150 & 0.0030963 & 0.0068288 & 0.0078554 \\
\hline \multirow{3}{*}{4} & 30 & 0.0231579 & 1.1713622 & 0.0860626 \\
\hline & 60 & 0.0091552 & 0.0918873 & 0.0583447 \\
\hline & 150 & 0.0052717 & 0.0097797 & 0.0194922 \\
\hline
\end{tabular}

Table (4)

IMSE values of the estimates of $R(t)$ for different sample sizes.

\begin{tabular}{|c|c|c|c|c|}
\hline Case & Sample sizes & $\widehat{R}(t)_{M L}^{N R}$ & $\widehat{R}(t)_{B S}^{L}$ & $\widehat{R}(t)_{B Q}^{L}$ \\
\hline \multirow{3}{*}{1} & 30 & 0.0044231 & 0.1891220 & 0.0600726 \\
\hline & 60 & 0.0033442 & 0.1375865 & 0.0599771 \\
\hline & 150 & 0.0029349 & 0.0019619 & 0.0598943 \\
\hline \multirow{3}{*}{2} & 30 & 0.0048832 & 0.0044912 & 0.0698393 \\
\hline & 60 & 0.0041619 & 0.0028614 & 0.0697503 \\
\hline & 150 & 0.0037947 & 0.0025382 & 0.0696669 \\
\hline \multirow{3}{*}{3} & 30 & 0.0085685 & 0.3069143 & 0.1078105 \\
\hline & 60 & 0.0071472 & 0.0074717 & 0.1077407 \\
\hline & 150 & 0.0070861 & 0.0060068 & 0.1075071 \\
\hline \multirow{3}{*}{4} & 30 & 0.0084504 & 122.271391 & 0.1207336 \\
\hline & 60 & 0.0080880 & 0.0787380 & 0.1206586 \\
\hline & 150 & 0.0073630 & 0.0063894 & 0.1203874 \\
\hline
\end{tabular}

